PROCEEDINGS OF THE

AMERICAN MATHEMATICAL SOCIETY

Volume 125, Number 9, September 1997, Pages 2521-2526

S 0002-9939(97)03836-7

\title{
INEQUIDIMENSIONALITY OF HILBERT SCHEMES
}

\author{
MEI-CHU CHANG
}

(Communicated by Wolmer V. Vasconcelos)

\begin{abstract}
We give a lower bound on the number of distinct dimensions of irreducible components of the Hilbert scheme of codimension 2 subvarieties in $\mathbb{P}^{n}$, for $n \leq 5$ (respectively, the moduli space of surfaces or 3 -folds) in terms of the Hilbert polynomial (resp. Chern numbers).
\end{abstract}

Let $\mathrm{Hilb}_{P}$ be the Hilbert scheme of subvarieties in the projective space with fixed Hilbert polynomial $P$ (respectively, let $\mathcal{M}$ be a moduli space of varieties with fixed Chern numbers). It is known that $\operatorname{Hilb}_{P}$ (resp. $\mathcal{M}$ ) has finitely many irreducible components and that the number of these components is bounded by some function of the Hilbert polynomial (resp. the Chern numbers). For work on the number of components of the Hilbert scheme (resp. the moduli space), see [EHM] for curves in $\mathbb{P}^{3}$ and [Ch1] for codimension 2 subvarieties in $\mathbb{P}^{n}$ with $n \leq 5$ (resp. [Ca1], [Ca2], [Ca3], [M] for surfaces and [Ch1] for surfaces and 3-folds).

The next question to ask is whether the Hilbert scheme (resp. moduli space) is equidimensional if it is reducible. Catanese [Ca3] has shown that for $\mathcal{M}$, the moduli space of surfaces, the number of distinct dimensions can be arbitrarily large.

In this note we study the number of distinct dimensions of the components of the Hilbert scheme $\operatorname{Hilb}_{P}$ (resp. moduli space $\mathcal{M}$ ) parametrizing subschemes with intersection numbers $H^{i} K^{n-2-i}$ (resp. Chern numbers), where $H$ is the hyperplane class and $K$ is the canonical class.

We define

$$
\begin{aligned}
n\left(d, H K, K^{2}\right)= & \#\{\operatorname{dim} H \mid H \text { is a component of the Hilbert scheme of } \\
& \text { surfaces in } \left.\mathbb{P}^{4} \text { with intersection numbers } d, H K, K^{2}\right\},
\end{aligned}
$$

$n\left(d, H^{2} K, H K^{2}, K^{3}\right)=\#\{\operatorname{dim} H \mid H$ is a component of the Hilbert schemes of 3-folds in $\mathbb{P}^{5}$ with intersection numbers $\left.d, H^{2} K, H K^{2}, K^{3}\right\}$, $n\left(K^{2}, c_{2}\right)=\#\{\operatorname{dim} H \mid H$ is a component of the moduli space of surfaces with Chern numbers $K^{2}, c_{2}$,

$n\left(K^{3}, c_{1} c_{2}, c_{3}\right)=\#\{\operatorname{dim} H \mid H$ is a component of the moduli space of 3 -folds with Chern numbers $\left.K^{3}, c_{1} c_{2}, c_{3}\right\}$.

Received by the editors October 5, 1995 and, in revised form, March 14, 1996.

1991 Mathematics Subject Classification. Primary 14J29; Secondary 14M07, 14M12.

Key words and phrases. Hilbert scheme, moduli space, projectively normal subvarieties, deformation theory, dimension.

The author was partially supported by NSF Grant No. DMS 9304580.

(C)1997 American Mathematical Society 
Notation. Let $d$ be a function of $r$. We write $d=O\left(r^{\alpha}\right)$ if there are positive constants $c_{1}$ and $c_{2}$ such that $c_{1} r^{\alpha} \leq d \leq c_{2} r^{\alpha}$ for $r$ sufficiently large.

What we can show is the following

Theorem. There is an infinite family of triples (respectively, quadruples) of integers $\left(d, H K, K^{2}\right)$ (resp. $\left(d, H^{2} K, H K^{2}, K^{3}\right)$ ) such that the Hilbert scheme of surfaces in $\mathbb{P}^{4}$ (resp. 3-folds in $\mathbb{P}^{5}$ ) with intersection numbers $d, H K, K^{2}$ (resp. d, $\left.H^{2} K, H K^{2}, K^{3}\right)$ has irreducible components of at least $O\left(y^{3 / 4}\right)($ resp. $O(y))$ distinct dimensions, where $y=K^{2}$ (resp. $\left.y=K^{3}\right)$.

In the statement above, replacing Hilbert scheme by moduli space and intersection numbers by Chern numbers, we have at least $O\left(y^{5 / 4}\right)$ many distinct dimensions for surfaces and $O(y)$ for 3-folds, $y$ being the self intersection number of the canonical class $K$. More precisely,

(i) For $K^{2} \gg 0$ there exist $d$ and $H K$ such that $n\left(d, H K, K^{2}\right) \geq O\left(\left(K^{2}\right)^{\frac{3}{4}}\right)$. Here $d=O\left(\left(K^{2}\right)^{\frac{1}{2}}\right)$ and $H K=O\left(\left(K^{2}\right)^{\frac{3}{4}}\right)$.

(ii) For $K^{3} \gg 0$ there exist $d=O\left(\left(K^{3}\right)^{\frac{2}{5}}\right), H^{2} K=O\left(\left(K^{3}\right)^{\frac{3}{5}}\right)$ and $H K^{2}=$ $O\left(\left(K^{3}\right)^{\frac{4}{5}}\right)$ such that $n\left(d, H^{2} K, H K^{2}, K^{3}\right) \geq O\left(\left(K^{3}\right)\right)$.

(iii) For $K^{2} \gg 0$ there exists $c_{2}=O\left(K^{2}\right)$ such that $n\left(K^{2}, c_{2}\right) \geq O\left(\left(K^{2}\right)^{\frac{5}{4}}\right)$.

(iv) For $K^{3} \gg 0$ there exist $c_{1} c_{2}$ and $c_{3}$ such that $n\left(K^{3}, c_{1} c_{2}, c_{3}\right) \geq O\left(\left(K^{3}\right)\right)$. Here $c_{1} c_{2}=O\left(\left(K^{2}\right)\right)$ and $c_{3}=O\left(\left(K^{2}\right)\right)$.

Remark 1. If $n(d, g)$ is the analogous notion for curves in $\mathbb{P}^{3}$ of degree $d$ and genus $g$, then for $g \gg 0$ there exists $d=O\left(g^{\frac{2}{3}}\right)$ such that $n(d, g) \geq O(g)$.

These results are obtained by studying the irreducible components corresponding to projectively normal subvarieties of codimension two in $\mathbb{P}^{n}$. Counting the number of distinct dimensions of such components boils down to the following

Problem. Let $c_{1} \leq \cdots \leq c_{r}$ be any increasing sequence of positive integers within a certain range. If the symmetric functions $s_{j}(\bar{c})=\sum_{i=1}^{r} c_{i}^{j}, j=1, \ldots, 5$, are fixed, how many values are taken on by the asymmetric, homogeneous function $t_{j}(\bar{c})=\sum_{i=1}^{5} i c_{i}^{j}$ or $u(\bar{c})=\sum_{i>j} c_{i}^{2} c_{j}$ ?

Our approach is to study the function $f=\left(s_{1}, \ldots, s_{5}, t_{1}, \ldots, t_{3}, u\right)$ defined on the positive real numbers. We show that the image of $f$ contains a "box" of maximal size. Hence the range of the fiber over a fixed $\left(s_{1}, \ldots, s_{5}\right)$ is as large as possible. Then we approximate the integral points in the fiber by the images of integral points (points with integral coordinates).

Question. Can one use the idea here and some formal deformation theory $[R]$ to obtain a much larger number of distinct dimensions of components for the moduli spaces? (See Remark 4 at the end of $\S 1$.)

The paper is organized in the following way. In section 1 we reduce our geometric question to a combinatorial one and prove the theorem. Section 2 is devoted to the combinatorial problem stated above. 
$\S 1$

Let $X$ be a projectively normal 3 -fold in $\mathbb{P}^{5}$ with resolution

$$
0 \rightarrow \underset{1}{\oplus} \mathcal{r + 2} \mathcal{O}\left(-a_{i}\right) \rightarrow \underset{-2}{\oplus} \mathcal{O}\left(-b_{j}\right) \rightarrow \mathcal{J}_{X} \rightarrow 0
$$

where $\left\{a_{i}\right\}$ and $\left\{b_{j}\right\}$ are two disjoint increasing sequences.

(A) (Ellingsrud [E]) Let $H$ be a component of the Hilbert scheme containing $X$. Then

$$
\begin{aligned}
\operatorname{dim} H=\sum_{a_{i} \geq b_{j}}\left(\begin{array}{c}
a_{i}-b_{j}+5 \\
5
\end{array}\right)+\sum_{b_{j} \geq a_{i}}\left(\begin{array}{c}
b_{j}-a_{j}+5 \\
5
\end{array}\right) \\
-\sum_{i \geq j}\left(\begin{array}{c}
a_{i}-a_{j}+5 \\
5
\end{array}\right)-\sum_{i \geq j}\left(\begin{array}{c}
b_{i}-b_{j}+5 \\
5
\end{array}\right)+1 .
\end{aligned}
$$

(B) Define

$$
\Delta_{k}:=\sum_{i} a_{i}^{k}-\sum_{j} b_{j}^{k}
$$

(a) Let $\Delta_{k}$ be of "degree" $k$. Then resolution (1) implies

(*) the Chern class $c_{i}\left(\mathcal{J}_{X}\right)$ is a degree- $i$ polynomial in the $\Delta$ 's.

In fact, $c_{2}\left(\mathcal{J}_{X}\right)=\frac{1}{2} \Delta_{2}, c_{3}\left(\mathcal{J}_{X}\right)=\frac{1}{3} \Delta_{3}, c_{4}\left(\mathcal{J}_{X}\right)=\frac{1}{4} \Delta_{4}+\frac{1}{8} \Delta_{2}^{2}$, and $c_{5}\left(\mathcal{J}_{X}\right)=$ $\frac{1}{5} \Delta_{5}+\frac{1}{6} \Delta_{2} \Delta_{3}$.

Let $a_{r+1}+a_{r+2}=29 r, b_{-2}=b_{-1}=b_{0}=10 r$, and

$$
\begin{aligned}
& a_{i}=b_{i}+1, i=1, \ldots, r, \\
& b_{i}=10 r+2 c_{i}, \quad \text { where } \quad r \leq c_{1} \leq c_{2} \leq \cdots \leq c_{r} \leq 2 r .
\end{aligned}
$$

(A result in [Ch1] implies that $X$ is nonsingular.)

(b) Combining (3), (4) and (5), we have

$$
\Delta_{j}=\sum_{h+k=j-1} s_{h}(\bar{c}) p_{k}(r)+p_{j}(r)
$$

where $s_{h}(\bar{c}):=\sum_{i} c_{i}^{h}$ and $p_{k}(r) \in \mathbb{Q}[r]$ is a polynomial of degree $k$, for $k=1, \ldots, j$.

In particular, we have

$$
\Delta_{j}=O\left(r^{j}\right)
$$

and

$$
c_{i}\left(\mathcal{J}_{X}\right)=O\left(r^{i}\right) .
$$

(c) Intersection theory gives

$$
c_{i}\left(\mathcal{J}_{X}\right)=H^{5-i}(K+6 H)^{i-2} .
$$

Combining (8) and (9), we have

$$
H^{3}=O\left(r^{2}\right), H^{2} K=O\left(r^{3}\right), H K^{2}=O\left(r^{4}\right), K^{3}=O\left(r^{5}\right) .
$$

(C) The intersection numbers $H^{i} K^{j}$ are functions of $r$ and $s_{j}(\bar{c})$. Indeed, this is a consequence of $(6),(*)$, and (9). 
With (C) in mind, we set the following

Notation. $A \equiv B$, if $A-B$ is a function of $r$ and $s_{j}(\bar{c})$. Here

$$
s_{j}(\bar{c})=\sum_{i} c_{i}^{j}
$$

(D) Substituting (4) and (5) into (2) and simplifying, we have

$$
\operatorname{dim} H \equiv \sum_{i>j}\left(\begin{array}{c}
2 c_{i}-2 c_{j}+4 \\
3
\end{array}\right) .
$$

So we reduce our problem to the following:

For $r \gg 0$, varying $r \leq c_{1} \leq \cdots \leq c_{r} \leq 2 r$ while $s_{j}(\bar{c})=\sum c_{i}^{j}$ being fixed, how many different values can $\sum_{i>j}\left(c_{i}-c_{j}\right)^{3}+2\left(c_{i}-c_{j}\right)$ or $2 \sum i c_{i}^{3}+6 \sum_{i>j} c_{i} c_{j}^{2}+2 \sum i c_{i}$ take?

Now part (ii) of the Theorem follows from (10) and a small variation of the following proposition which will be proved in the next section.

Proposition. Let $A=A_{\left(s_{1}, \ldots, s_{5}\right)}=\left\{\left(c_{1}, \ldots, c_{r}\right) \mid r \leq c_{1} \leq \cdots \leq c_{r} \leq 2 r, c_{i} \in \mathbb{N}\right.$, $s_{j}(\bar{c}):=\sum c_{i}^{j}=s_{j}$ for $\left.j=1, \ldots, 5\right\}$, and let $g=\left(g_{1}, \ldots, g_{4}\right): A \rightarrow \mathbb{N}^{4}$ be defined by $g_{j}(\bar{c})=\sum i c_{i}^{j}$ for $j=1,2,3$ and $g_{4}(\bar{c})=\sum_{i>j} c_{i}^{2} c_{j}$. Then for $r \gg 0$, there is a box $B(r)$ of size $r^{2} \times \cdots \times r^{6}$ in $\mathbb{N}^{5}$ such that for any $S=\left(s_{1}, \ldots, s_{5}\right)$ in $B(r)$, there is a box $B(S)$ in $\mathbb{N}^{4}$ of size $r^{3} \times r^{4} \times r^{5} \times r^{5}$ such that the image of $g$ contains $B(S)$.

Remark 2. Exactly the same construction for surfaces in $\mathbb{P}^{4}$ gives $H^{2}=O\left(r^{2}\right), H K$ $=O\left(r^{3}\right), K^{2}=O\left(r^{4}\right)$ for $(10)$, and $\operatorname{dim} H \equiv \sum_{i>j}\left(\begin{array}{c}2 c_{i}-2 c_{j}+3 \\ 2\end{array}\right)$ for (11), hence part (i) of the Theorem.

Remark 3. In [Ch2] where we showed the number of components of the Hilbert schemes of some of such projectively normal 3 -folds $X$ is at least $y^{\left(y^{\frac{1}{5}}\right)}$, where $y=K^{3}$, we have seen that the line bundle $\mathcal{O}_{X}(1)$ and its sections deform along with $X$. Also a quintic hypersurface section $Y=X \cap 5 H$ deforms along with $X$. These give parts (iii) and (iv) of the Theorem.

Remark 4. It is plausible that one could show $n\left(K^{2}, c_{2}\right)$ and $n\left(K^{3}, c_{1} c_{2}, c_{3}\right)$ can be arbitrarily large (in terms of the Chern numbers) by considering $Y$ to be the intersection of suitably many hypersurfaces with a projectively Cohen-Macaulay subvariety $X$ of codimension 2 in $\mathbb{P}^{n}$ for odd $n \gg 0$, where the ideal defining $X$ has a similar resolution to the defining ideal of the variety considered here. The same combinatorics show that the number of distinct dimensions of components of Hilbert scheme parametrizing subvarieties of $\mathbb{P}^{n}$ with the same Hilbert polynomial as $X$ is in the order of $r^{n}$, while [Ch1] implies that the singularity of $X$ has expected dimension (hence $Y$ is nonsingular) and the adjunction formula implies that $K_{Y}^{2}=O\left(r^{4}\right)$ and $c_{2}(Y)=O\left(r^{4}\right)$.

In this section, we will prove the Proposition stated in $\S 1$. Our approach is to study the function $f=\left(s_{1}, \ldots, s_{5}, g_{1}, \ldots, g_{4}\right)$ rather than the sequence $\left(g_{1}, \ldots, g_{4}\right)$. After showing that the image of $f$ contains a large enough box, we study the fibers over a point $\left(s_{1}, \ldots, s_{5}\right)$. For this study, it is more natural to first consider $f$ as 
a function of continuous functions (rather than sequences, which are just discrete functions on a finite set), and then to approximate the integral points in the images of integral points.

Hence the following lemma is the key.

Lemma 1. Let $C^{1}$ be the set of continously differentiable functions, and let $F=$ $\left(F_{1}, \ldots, F_{9}\right): C^{1} \rightarrow \mathbb{R}^{9}$ be an operator defined by

$$
F_{j}(\varphi)=\left\{\begin{array}{l}
\int_{r}^{2 r} \varphi^{j}(x) d x, j=1, \ldots, 5, \\
\int_{r}^{2 r} x \varphi^{j-5}(x) d x, j=6,7,8, \\
\int_{2 r \geq x>y \geq r} \varphi^{2}(x) \varphi(y) d x d y, j=9 .
\end{array}\right.
$$

Then for any $K \gg 0$, there is a set $S$ consisting of increasing functions, and a box $Q_{K}$ in $\mathbb{R}^{9}$ of size $d_{1} r K \times \cdots \times d_{5} r K^{5} \times d_{6} r^{2} K \times \cdots \times d_{8} r^{2} K^{3} \times d_{9} r^{2} K^{3}$, where $d_{i}$ 's are constants independent of $r$ and $K$ such that $F(S) \supset Q_{K}$.

Proof. Define $f: C^{1} \rightarrow \mathbb{R}^{9}$ by

$$
\begin{aligned}
f(\varphi)=\left(\int_{1}^{2} \varphi d x, \ldots, \int_{1}^{2} \varphi^{5} d x, \int_{1}^{2} x \varphi d x, \ldots\right. \\
\left.\qquad \int_{1}^{2} x \varphi^{3} d x, \int_{2 \geq x>y \geq 1} \varphi^{2}(x) \varphi(y) d x, d y\right) .
\end{aligned}
$$

Let $D_{\psi} f_{j}(\varphi)=\lim _{t \rightarrow 0} \frac{f_{j}(\varphi+t \psi)-f_{j}(\varphi)}{t}$ be the directional derivative of $f_{j}$ in the direction of $\psi$ at $\varphi$.

$(\alpha)$ If $D_{\psi} f_{j}(\varphi)=\left\langle\nabla f_{j}(\varphi), \psi\right\rangle=\int \nabla f_{j}(\varphi) \cdot \psi$, where $\nabla f_{j}$ is the gradient of $f_{j}$ identified in the dual space, and

$(\beta)$ if there is $\varphi$ such that $\nabla f_{1}(\varphi), \ldots, \nabla f_{9}(\varphi)$ are linearly independent (these are $j \varphi^{j-1}$ for $j=1, \ldots, 5, x j \varphi^{j-1}$ for $j=6,7,8$ and $\nabla f_{9}(\varphi)=2 \varphi \int_{x}^{2} \varphi(y) d y+$ $\left.\int_{1}^{2} \varphi^{2}(y) d y\right)$, then there are $\psi_{1}, \ldots, \psi_{9}$ such that

$$
D_{\psi_{i}} f_{j}(\varphi)=\delta_{i j}
$$

Now define $\bar{f}: \mathbb{R}^{9} \rightarrow \mathbb{R}^{9}$

$$
\bar{f}_{j}\left(t_{1}, \ldots, t_{9}\right)=f_{j}\left(\varphi+t_{1} \psi_{1}+\cdots+t_{9} \psi_{9}\right) .
$$

(Note that $\bar{f}$ factors through $C^{1}$ via the map $g: \mathbb{R}^{9} \rightarrow C^{1}$ defined by

$$
g\left(t_{1}, \ldots, t_{9}\right)=\varphi+t_{1} \psi_{1}+\cdots+t_{9} \psi_{9}
$$

i.e. we have $\bar{f}_{j}=f_{j} \circ g$.)

It is easy to see that

$$
\frac{\partial \bar{f}_{j}}{\partial t_{i}}(0)=D_{\psi_{i}} f_{j}(\varphi)=\delta_{i j}
$$

So the implicit function theorem implies that the image of $\bar{f}$ contains a ball $\bar{B}$, say, of size $d_{1} \times \cdots \times d_{9}$ in $\mathbb{R}^{9}$. Let $B$ be a ball in $\mathbb{R}^{9}$ that contains the preimage of $\bar{B}$, i.e. $\bar{f}(B) \supset \bar{B}$.

To satisfy conditions $(\alpha)$ and $(\beta)$, we take $\varphi=\sin \frac{x}{10}$. To make sure $Q_{K}$ is mapped by increasing functions, we take $\psi_{i}$ 's such that $\frac{d \psi_{i}}{d x}$ is much smaller than $\frac{d \varphi}{d x}$. The set $S$ is $g(B)$. Clearly enlarging the ball $B$ by $K$ (i.e. letting $\bar{\varphi}=K \varphi$ ) gives the image of size $d_{1} K \times \cdots \times d_{5} K^{5} \times d_{6} K \times \cdots \times d_{8} K^{3} \times d_{9} K^{3}$, and rescaling (i.e. letting $\bar{x}=r x$ ) gives the factor $r$ or $r^{2}$ in each of the coordinates. 
Lemma 2. Let $B_{r}=\left\{\left(c_{\frac{r}{2}+1} \ldots, c_{r}\right) \mid r \leq c_{\frac{r}{2}+1} \leq \cdots \leq c_{r} \leq 2 r, c_{i} \in \mathbb{N}\right\}$ and $f: B_{r} \rightarrow$ $\mathbb{N}^{9}$ defined by $f=\left(s_{1}, \ldots, s_{5}, t_{1}, \ldots, t_{3}, u\right)$, where $s_{j}(\bar{c})=\sum c_{i}^{j}, t_{j}(\bar{c})=\sum i c_{i}^{j}$, and $u(\bar{c})=\sum_{i>j} c_{i}^{2} c_{j}$. Then there is a box $Q_{r}$ of size $r^{2} \times \cdots \times r^{6} \times r^{3} \times r^{4} \times r^{5} \times r^{5}$ in $\mathbb{N}^{9}$ such that every integral point in $Q_{r}$ lies together with the image under $f$ of an integral point of $B_{r}$, in a box of size $r \times \cdots \times r^{5} \times r^{2} \times r^{3} \times r^{4} \times r^{4}$.

Proof. In Lemma 1, take $c_{\frac{r}{2}+k}=[\varphi(r+2 k)]$, the integral part of $\varphi(r+2 k)$.

Lemma 3. Let $A_{r}=\left\{\left(c_{1}, \ldots, c_{r}\right) \mid r \leq c_{1} \leq \cdots \leq c_{r} \leq 2 r, c_{i} \in \mathbb{N}\right\}$ and $f$ be as in Lemma 2. Then for $r \gg 0$, there is a box $Q_{r}$ of size $r^{2} \times \cdots \times r^{6} \times r^{3} \times r^{4} \times r^{5} \times r^{5}$ such that every integral point in $Q_{r}$ is approximated by the image of an integral point up to arbitrarily small power of $r$.

Proof. Take $r_{1}=r, r_{2}, \ldots$ such that $r_{i}>r_{i+1}>r_{i}^{\frac{5}{6}}$ and apply Lemma 2 for each $r_{i}$, then take the Cartesian product of all $B_{r_{i}}$ 's. (Note: if $(\bar{c}, \bar{d}) \in B_{r_{i}} \times B_{r_{k}}$ for $i>k$, then $s_{j}(\bar{c}, \bar{d})=s_{j}(\bar{c})+s_{j}(\bar{d}) \quad u(\bar{c}, \bar{d})=u(\bar{c})+u(\bar{d})+s_{2}(\bar{c}) s_{1}(\bar{d}), t_{j}(\bar{c}, \bar{d})>$ $\left.t_{j}(\bar{c})+t_{j}(\bar{d}).\right)$

To conclude the proof of the Proposition, we notice that $\left.A_{(} s_{1}, \ldots, s_{5}\right)$ is the fiber of $A_{r}$ in Lemma 3 over a point $\left(s_{1}, \ldots, s_{5}\right)$.

\section{ACKNOWLEDGEMENT}

We would like to thank the referee for helpful comments which made the paper more readable. We specially thank Scott Nollet for a very careful reading and many helpful editorial discussions to have the paper in the present form.

\section{REFERENCES}

[Ca1] F. Catanese, Chow varieties, Hilbert schemes, and moduli spaces of surfaces of general type,, Journal of Algebraic Geometry 1 (1992), 561-595. MR 93j:14005

[Ca2] F. Catanese, Connected components of moduli spaces, Journal of Differential Geometry 24 (1986), 395-399. MR 87m:14036

[Ca3] F. Catanese, On the moduli space of surfaces of general type, Journal of Differential Geometry 19 (1984), 483-513. MR 86h:14031

[Ch1] M. Chang, A filtered Bertini-type theorem, J. reine angew. Math. 397 (1989), 214-219. MR 90i: 14054

[Ch2] M. Chang, The number of components of Hilbert schemes, preprint. CMP 96:14

[E] P. Ellingsrud, Sur le schéma de Hilbert des variétés de codimension 2 dans $\mathbb{P}^{e}$ a cône de Cohen-Macaulay, Ann. scient. Éc. Norm. Sup. $4^{\circ}$ série, t. 8 (1975), 423-432. MR 52:13831

[EHM] Ph. Ellia, A. Hirschowitz, and E. Mezzetti, On the number of irreducible components of the Hilbert schemes of smooth space curves, International Journal of Math 3 (6) (1992), 799-807. MR 93j:14006

[M] M. Manetti, Iterated double covers and connected components of moduli spaces, Preprint, 1994.

[R] Z. Ran, Deformation of maps, Algebraic Curves and Projective Geometry, Lecture Notes in Math., Springer-Verlag 1389 (1989), 245-253. MR 91f:32021

School of Mathematics, Institute for Advanced Study, Princeton, New Jersey 08540 E-mail address: mcc@math.ias.edu

Current address: Department of Mathematics, University of California, Riverside, California 92521

E-mail address: mcc@math.ucr.edu 